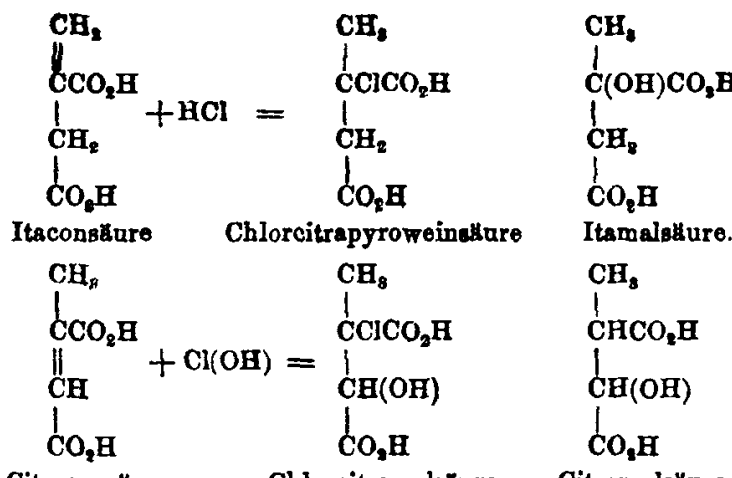

Citraconsture Chlorcitramalaxure

Citramalehare.

Im letzteren Falle addirt sich die Hydroxylgruppe der unterchlorigen Säure, als die negativere, zu demjenigen Kohlenstoffatom, welches sich unter dem gröfsten Einflusse der negitiven Elemente des Moleculs befindet. Die aufgestellten Formeln müssen jedenfalls als vorläufige betrachtet werden, da sowohl bei der Bildung der Chlorpyroweinsäure als auch der Chlorcitramalsäure die Möglichkeit der gleichzeitigen Bildung von Isomeren nicht ausgeschlossen ist, da die Anlagerung der Elemente des $\mathrm{HCl}$ und $\mathrm{Cl}(\mathrm{OH}$ ) auch in entgegengesetzter Ordnung stattfinden kann. Unsere Kenntnisse über die Bedingungen, bei welcher diese Additionsproducte auftreten, sowie auch die Umwandlungen derselben sind zur Zeit noch nicht hinreichend abgeschiossen.

III. Ueber die Darstellung von Trimethylenbromid; von $J$. Lermontoff.

Die Darstellung von Trimethylenbromid ist schon vielfach Gegenstand der Untersuchung gewesen. Aus den Arbeiten 
von Geromont und Reboul ist es bekannt, dafs diese Verbindung sich bei directer Einwirkung von rauchender Bromwasserstoffsaure auf Allylbromid bildet, aber stets mit Beimengungen betrāchtlicher Mengen des mit ihm isomeren Propylenbromids. Später giebt Ka y s e r, der sich mit diesem Gegenstand sehr viel befafst hat, eine Methode, nach welcher er ganz reines Trimethylenbromid erhalten zu haben glaubt.

Gelegentlich der Arbeit von H. Markownik of f wurde ich auf die Darstellung des Trimethylenbromids geführt. Es schien mir nicht ohne Interesse zu sein, in diesem Falle den Einflufs der Temperatur sowie auch von anderen Bedingungen auf die Bildung dieser isomeren Verbindungen etwas genauer zu erforschen. Zu diesem Zwecke stellte ich eine ganze Reihe von Versuchen an, deren Resultate ich hier mittheile. Ich brachte zuerst die von R e boul und Kaysser gegebenen Methoden in Anwendung, aber durch keine derselben konnte ich zu einigermalsen ergiebigen Resultaten kommen. Rebou l giebt an, es genüge, Allylbromid mit rauchender Bromwasserstoffsäure kurze Zeit auf $100^{\circ}$ zu erhitzen, um neben kleiner Menge unveränderten Bromids $\% / 3$ Trimethylenbromid und $1 / 3$ Propylenbromid zu erhalten. Bei Wiederholung dieses Versuches habe ich das Erhitzen unter denselben Bedingungen acht Tage lang fortdauern lassen, wobei die Gefäfso von Zeit zu Zeit aufgeschmolzen wurden und die Flüssigkeit von Neuem mit Bromwasserstofigas gesättigt wurde. Dabel blieb jedoch immer eine nicht geringe Menge Allylbromid unverändert und von dem hochsiedenden Antheil der Bromide ergab sich $3 / 6$ als Propylenbromid und nur $1 / 4$ als Trimethylenbromid.

Diese Resultate liefsen mich vermuthen, dafs für die erwũnschte Bildung des Trimethylenbromids die Temperatur nicht hoch genug gewesen war. Es lagen aber die Angaben von Kaysser vor, die solcher Vermuthung vollständig wider- 
sprachen: denn nach seiner Reschreibung erhielt er reines Trimethylenbromid bei etwa der Siedctemperatur des Allylbromids, d. h. gegen $71^{\circ}$.

Seine sehr ausführlich beschriehenen Versuche wurden nit der gröfsten Sorgfalt wiederholt. $100 \mathrm{Grm}$. Allylbromid wurden in einem constanten Strome trockener Bromwasserstoffsäure ganz langsam destillirt. Die Destillation dauerte eine Stunde, welche Operation acht Mal hintereinander wiederholt wurde und zwar so, dafs das Destillat immer wieder destillirt wurde. Auf diese Weise erhielt ich nur ganz geringe Spuren eines hochsiedenden Products, das im Wasserbade nicht übergehen wollte. Obgleich dieser Versuch einige Mal genau mit dem von Kays s er beschriebenen Apparate wiederholt wurde, gelang es mir nie, auch nur $1 / 10$ der angewandten Menge eines Products zu erhalten, welches ich als Trimethylenbromid erkennen konnte.

Der oben beschriebene Versuch wurde dann auf die Weise ungeändert, dafs Allylbromid in einem Strome trockener Bronıwasserstoffsäure in einem Kolben mit aufrechtstehendem Kühler 6 bis 7 Tage digerirt wurde. Hierbei hatte ich keinen besseren Erfolg. In der Hoffnnng, ein günstigeres Resultat zu erlangen, erhitzte ich mit (durch Phosphorsäureanhydrid) getrockneter Bromwasserstoffsäure gesältigte Dämpfe von Allylbromid, vor der Condensation auf etwa 110 bis $120^{\circ}$. Die Einrichtung bestand aus einem langen Liebig'schen Kühler, welcher durch die Dämpfe von Amylalkobol erhitzt wurde, während durch das innere Rohr die mit Allylbromid gesättigte Bromwasserstoffsäure langsam strich. Die Destillation von $100 \mathrm{Grm}$. Allylbromid liefs ich hier vier Stunden dauern, aber trotzdem war das Condensationsproduct nichts als unverändertes Allylbromid. Aus diesen Versuchen kam ich zu der Ueberzeugung, dafs die Gegenwart von Wasser auf die Verbindungsfähigkeit des Bromwasserstoffs von grofser 
Bedeutung ist, und dafs für eine vollständige Umwandlung des Allylbromids in Trimethylenbromid eine weit höhere Temperatur erforderlich sei. Der Versuch bestätigte meine Vermuthung, was auch mit der von H. Markownik off ausgesprochenen Gesetzmärsigkeit im vollkornmenen Einklange steht *). Er äufserte die Meinung, dafs die Temperatur der Körper die Richtung bedingt, in welcher dieselben auf einander chemische Wirkung ausüben können.

Ich verfuhr auf folgende Weise : Allylbromid wurde in dem Gefäfse, in das as später eingeschmolzen werden sollte, bei einer Temperatur von $-10^{\circ}$ bis $-15^{\circ}$ mit trockenem Bromwasserstoffgas gesälliugt. Bei niedriger Temperalur absorbirt das Allylbromid ziemlich viel Bromwasserstoff. Das Erhitzen wurde bei einer Temperatur von 165 bis $170^{\circ}$ ausgeführt und dauerte 24 Stunden. Dabei ergab sich die ganze Masse des hochsiedenden Products als fast vollstãndig reines Trimethylenbromid, dem nur ein geringer Antheil Propylenbromid beigemengt war, während $1 / 9$ des Allylbromids unverbunden blieb. Für die Umwandlung der ganzen Menge des angewendeten Allylbromids genügt es die Flüssigkeit noch einmal mit Bromwasserstoffsäure zn sättigen und wieder 24 Stunden lang zu erhitzen. Das Reactionsproduct ist dunkelbraun gefärbt und wurde zur Reinigung mit Wasserdämpfen destillirt.

Da die Versuche in Glasgefäfsen angestellt wurden, war es unmöglich, ohne Gefahr von Explosion die Temperatur höher zu steigern, wodurch die auch geringe Beimengung von gewöhnlichem Propylenbromid hätte vermieden werden können; denn bei meinen melırfach bei verschiedenen Temperaturen wiederholten Versuchen beobachtete ich stets, dafs

*) Compt. rend. 1875 . 
die Menge des Propylenbromids mit Erniedrigung der Temperatur steigt.

Nach diesem von mir beschriebenen Verfahren wäre es möglich, ohne grofse Gefahr eine beliebige Menge von Trimethylenbromid in verhältnifsmäfsig kurzer Zeil darzustellen, wenn man nur die dazu passenden Gefäfse finden könnte.

\section{Das Aceton im Harne der Diabetiker;}

$$
\text { von W. Markownikoff. }
$$

Das Aceton, diese schon seit langer Zeit bekannte organische Verbindung, ist erst seit der letzten Zeit als Educt des thierischen Organismus in einigen pathologischen Fällen beobachtet worden. Das Auftreten desselben im Harne der Diabetiker wurde in den Fällen wahrgenommen, wo sich der gawōhnliche Diabetes durch besondere, noch nioht hinreichend scharf bezeichnete Erscheinungen complicirte, und welche die neuere Medicin mit dem Namen Acetonourie charakterisirt. Die Zahl der Fälle, wo Aceton unzweifelhaft nachgewiesen wurde, ist aber eine sehr beschränkte. Un die Ursache der Acetonomie aufuklären und ihren Zusanmenhang mit dem Diabetes festzustellen, ist es unbedingt nöthig, die Gegenwart des Acetons im Harne in den Fällen nachzuweisen, wenn die den Diabetes modificirenden Synptome noch sehr schwach oder noch nicht vorhanden sind. Im vorigen und in diesem Jahre untersuchte ich den Harn zweier Diabetiker aus der Universitätsklinik. Um Aceton zu erhalten, unterwarf man den in 24 Stunden gesammelten, mit Weinsäure angesäuerten Harn einer systematischen Destillation von $1 / 2$ bis $2 / 8$ des anfanglichen Volums. Nach den drei ersten Destillationen wurde 